\title{
A New Cycloartane-Type Triterpenoid Saponin Xanthine Oxidase Inhibitor from Homonoia riparia Lour
}

\author{
Fan Xu ${ }^{1}$, Xueqian Zhao ${ }^{1}$, Lingli Yang ${ }^{2}$, Xiuhua Wang ${ }^{1}$ and Jing Zhao ${ }^{1, *}$ \\ 1 Kunming General Hospital of Chengdu Military Region, Kunming 650032, Yunnan, China; \\ E-Mails: xu_fan@126.com (F.X.); zhaoxueqian@126.com (X.Z.); wangxh2341@126.com (X.W.) \\ 2 The First Affiliated Hospital of Kunming Medical University, Kunming 650031, Yunnan, China; \\ E-Mail: lingli_yang@126.com \\ * Author to whom correspondence should be addressed; E-Mail: xu_fan2014@163.com; \\ Tel.: +86-871-6477-4308; Fax: +86-871-6531-6752.
}

Received: 30 June 2014; in revised form: 6 August 2014 / Accepted: 7 August 2014 /

Published: 29 August 2014

\begin{abstract}
A new cycloartane-type triterpenoid saponin named riparsaponin (1) was isolated from the stem of Homonoia riparia Lour together with six known compounds. The structure of riparsaponin was determined by using NMR and mass spectroscopy and X-ray crystallography techniques. Additionally, riparsaponin has a significant inhibitory effect on xanthine oxidase in vitro, and the $\mathrm{IC}_{50}$ was $11.16 \mathrm{nmol} / \mathrm{mL}$.
\end{abstract}

Keywords: Homonoia riparia Lour; cycloartane-type triterpenoid saponin; xanthine oxidase inhibitors

\section{Introduction}

Homonoia riparia Lour, (family Euphorbiaceae), is widely distributed in the south part of China [1]. The roots of $H$. riparia are commonly used as an effective traditional Chinese herbal medicine for treating hepatitis and joint gall, stomach ache, and ambustion based on its antipyretic choleretic, anti-inflammatory, detoxification, and diuretic activities [1,2]. However, phytochemical and pharmacological investigations of this plant are currently lacking. Previous chemical research reported that it contains triterpenes, steroids, and phenolics [3-5]. In our present investigation, a new cycloartane-type triterpenoid saponin (compound 1, named riparsaponin) was isolated from the stems of $H$. riparia together with six known compounds (Figure 1). In addition, riparsaponin showed 
significant inhibitory activity on xanthine oxidase. Here, we report the isolation, identification and activity of the new compound, which could be helpful for treating gouty arthritis.

Figure 1. Structure of the compounds isolated from H. riparia.

<smiles>C/C=C/C=C/C(=O)O</smiles><smiles>CC(C)OC1CCC2(C)C(CCC1(C)C)C1=CCC3(C(=O)O)CCC(C)(C)C2CCC13C</smiles>

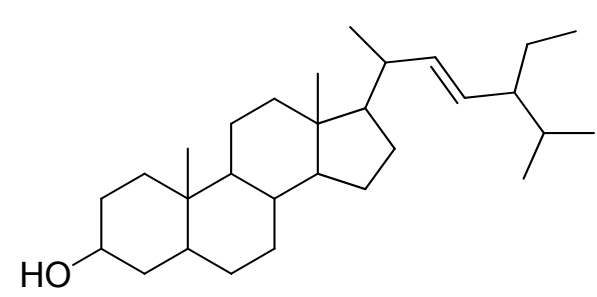

5

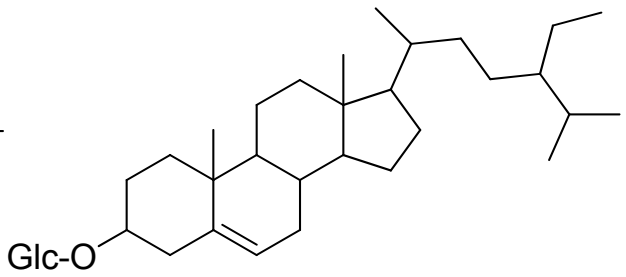

6

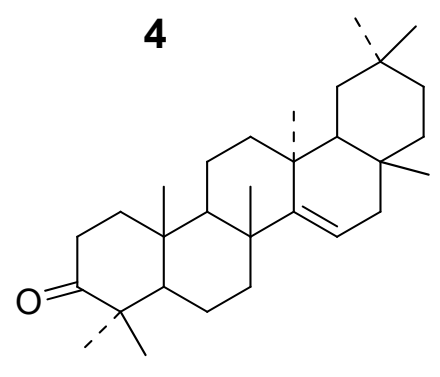

7

\section{Results and Discussion}

\subsection{Identification of the Riparsaponin}

The new compound riparsaponin (1) was identified by using NMR and mass spectroscopy, and X-ray crystallography techniques. Compound 1 was obtained as colorless prismatic crystal $(\mathrm{MeOH})$; mp: 291-292 ${ }^{\circ} \mathrm{C}$. Its Liebermann-Burchard response was positive. FAB-MS (m/z) (\%): 620 (99), 712 (29), 487 (55). The ${ }^{13} \mathrm{C}-\mathrm{NMR},{ }^{1} \mathrm{H}-\mathrm{NMR}$ data are listed in Table 1 . The ${ }^{13} \mathrm{C}-\mathrm{NMR}$ spectrum $(100 \mathrm{MHz}$, DMSO- $\left.d_{6}\right)$ showed 36 carbon signals, and lots of carbon signals were between $60-80\left(\delta_{\mathrm{C}}\right)$, which indicated the existence of a saccharide group. The carbon signals of 105.9, 73.82, 105.9, 73.82, and $65.62\left(\delta_{\mathrm{C}}\right)$ could further demonstrate the existence of pentose, and the carbon signal at $105.9 \mathrm{ppm}$ is the terminal group carbon of the pentose ( $\beta$-type) [6]. The two high-field doublets observed at $\delta_{\mathrm{H}} 0.99$ $(1 \mathrm{H}, \mathrm{m})$ and $0.3(1 \mathrm{H}, \mathrm{s})$ are characteristic of the two germinal protons of a cyclopropane moiety. What's more, two olefinic protons at $\delta_{\mathrm{H}} 4.65(2 \mathrm{H}, \mathrm{brs})$ indicated the presence of an olefinic methylene moiety. There are two carbon signals at 106.13 and $156.22\left(\delta_{\mathrm{C}}\right)$, suggesting a double bond. The ${ }^{1} \mathrm{H}-\mathrm{NMR}$ spectrum (400 MHZ, DMSO- $\left.d_{6}\right)$ indicated seven methyl groups $\left(\delta_{\mathrm{H}} 0.87,0.88,0.96,0.98\right.$, $1.05,1.08,1.12)$. These spectroscopic data suggested this compound to be a cycloartane-type triterpene with an exocyclic methylene group. Combined the reference data [3], we found the ${ }^{1} \mathrm{H}-\mathrm{NMR}$ and ${ }^{13} \mathrm{C}$-NMR spectra of compound $\mathbf{1}$ were analogous to that reported for a cycloartane-type triterpene which was isolated from $H$. riparia previously. Furthermore, the structure of compound $\mathbf{1}$ was confirmed by HMBC, ROESY (Figure 2) and X-ray diffraction (CCDC deposition number is 1021164) (Figure 3, Table 2). 
Figure 2. The key HMBC (a) and ROESY (b) connections of riparsaponin (1).

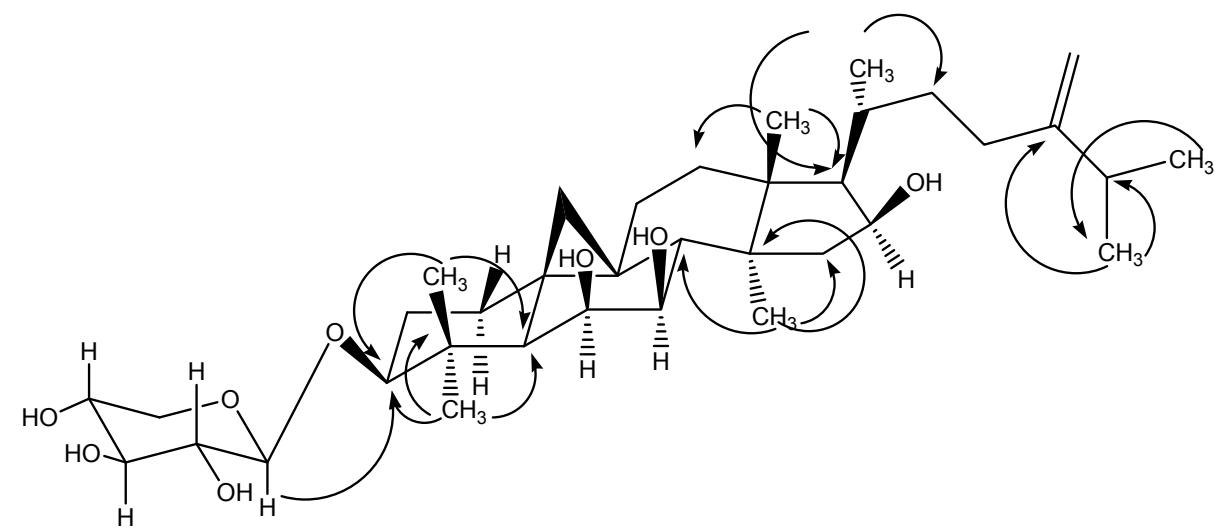

(a)

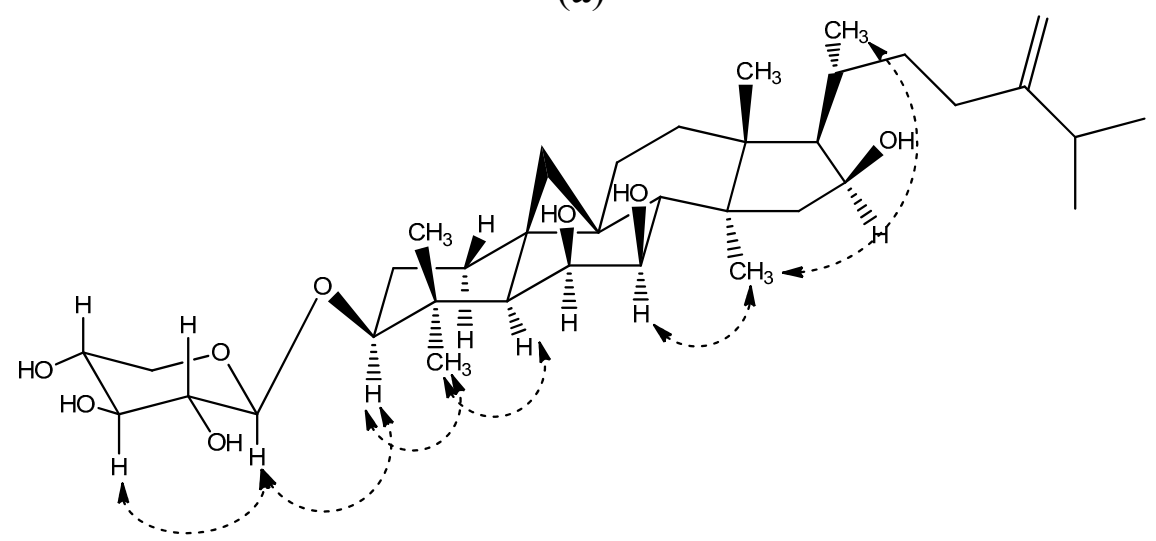

(b)

Figure 3. Perspective drawing of compound 1 generated from X-ray crystal data.

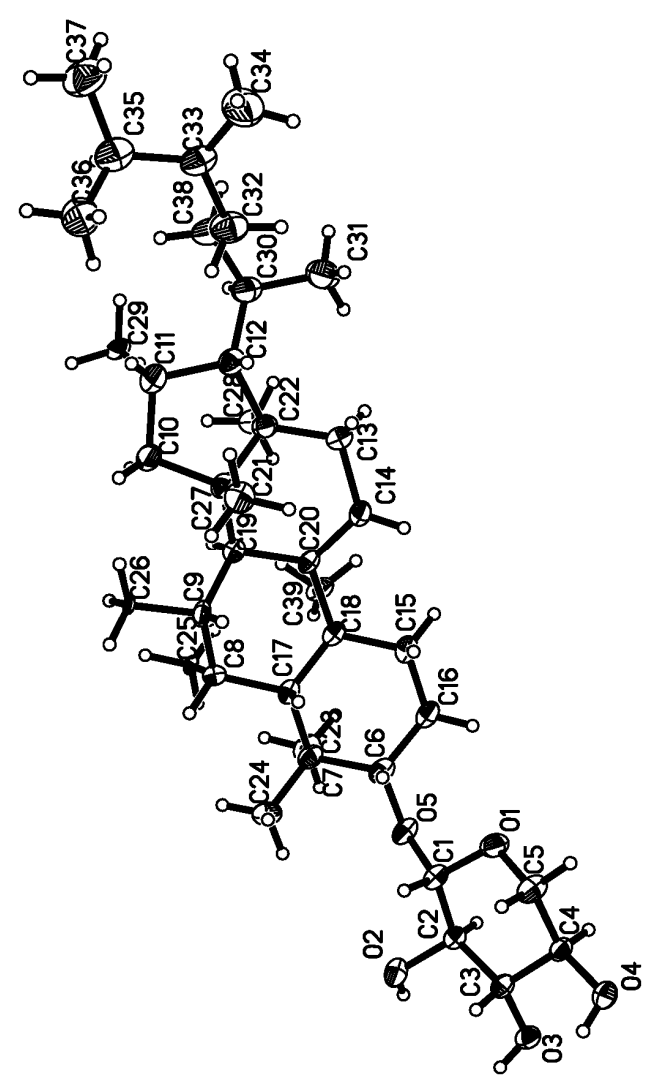


Table 1. ${ }^{1} \mathrm{H}-\mathrm{NMR}(400 \mathrm{~Hz})$ and ${ }^{13} \mathrm{C}-\mathrm{NMR}(100 \mathrm{~Hz})$ data of riparsaponin in DMSO- $d_{6}$.

\begin{tabular}{|c|c|c|c|c|c|}
\hline & $\boldsymbol{\delta}_{\mathrm{C}}$ & $\delta_{H}$ & & $\boldsymbol{\delta}_{\mathrm{C}}$ & $\delta_{\mathrm{H}}$ \\
\hline 1 & $32.62 \mathrm{t}$ & $1.53(2 \mathrm{H}, \mathrm{m})$ & 19 & $31.41 \mathrm{t}$ & $\begin{array}{c}0.99(1 \mathrm{H}, \mathrm{m}) \\
0.30(1 \mathrm{H}, \mathrm{s})\end{array}$ \\
\hline 2 & $29.16 \mathrm{t}$ & $\begin{array}{l}1.72(1 \mathrm{H}, \mathrm{m}) \\
1.53(1 \mathrm{H}, \mathrm{m})\end{array}$ & 20 & $29.77 \mathrm{~d}$ & $1.77(1 \mathrm{H}, \mathrm{m})$ \\
\hline 3 & $87.52 \mathrm{~d}$ & $3.00(1 \mathrm{H}, \mathrm{m})$ & 21 & $17.92 \mathrm{q}$ & $0.87(3 \mathrm{H}, \mathrm{d}, J=8.6 \mathrm{~Hz})$ \\
\hline 4 & $40.82 \mathrm{~s}$ & & 22 & $34.54 \mathrm{t}$ & $\begin{array}{l}1.80(1 \mathrm{H}, \mathrm{m}) \\
1.08(1 \mathrm{H}, \mathrm{m})\end{array}$ \\
\hline 5 & $49.02 \mathrm{~d}$ & $1.22(1 \mathrm{H}, \mathrm{s})$ & 23 & $31.35 \mathrm{t}$ & $\begin{array}{l}2.07(1 \mathrm{H}, \mathrm{m}) \\
1.88(1 \mathrm{H}, \mathrm{m})\end{array}$ \\
\hline 6 & $71.38 \mathrm{~d}$ & $3.74(1 \mathrm{H}, \mathrm{brs})$ & 24 & $156.22 \mathrm{~s}$ & \\
\hline 7 & $72.74 \mathrm{~d}$ & $3.21(1 \mathrm{H}, \mathrm{m})$ & 25 & $33.15 \mathrm{~d}$ & $2.22(1 \mathrm{H}, \mathrm{m})$ \\
\hline 8 & $45.90 \mathrm{~d}$ & $1.88(1 \mathrm{H}, \mathrm{d}, J=11.2 \mathrm{~Hz})$ & 26 & $21.76 \mathrm{q}$ & $0.96(3 \mathrm{H}, \mathrm{s})$ \\
\hline 9 & $24.04 \mathrm{~s}$ & & 27 & $21.81 \mathrm{q}$ & $0.98(3 \mathrm{H}, \mathrm{s})$ \\
\hline 10 & $18.72 \mathrm{~s}$ & & 28 & $19.43 \mathrm{q}$ & $0.88(3 \mathrm{H}, \mathrm{s})$ \\
\hline 11 & $25.43 \mathrm{t}$ & $\begin{array}{l}1.91(1 \mathrm{H}, \mathrm{m}) \\
0.92(1 \mathrm{H}, \mathrm{m})\end{array}$ & 29 & $24.07 \mathrm{q}$ & $1.05(3 \mathrm{H}, \mathrm{s})$ \\
\hline 12 & $32.27 \mathrm{t}$ & $\begin{array}{l}1.36(1 \mathrm{H}, \mathrm{m}) \\
1.08(1 \mathrm{H}, \mathrm{m}) \\
\end{array}$ & 30 & $16.40 \mathrm{q}$ & $1.08(3 \mathrm{H}, \mathrm{s})$ \\
\hline 13 & $45.64 \mathrm{~s}$ & & 31 & $106.13 \mathrm{t}$ & $4.65(2 \mathrm{H}, \mathrm{brs})$ \\
\hline 14 & $45.41 \mathrm{~s}$ & & $\mathbf{1}^{\prime}$ & $105.90 \mathrm{~d}$ & $4.09(1 \mathrm{H}, \mathrm{d}, J=7.5 \mathrm{~Hz})$ \\
\hline 15 & $51.66 \mathrm{t}$ & $\begin{array}{l}2.16(1 \mathrm{H}, \mathrm{m}) \\
1.41(1 \mathrm{H}, \mathrm{m}) \\
\end{array}$ & $\mathbf{2}^{\prime}$ & $73.82 \mathrm{~d}$ & $2.95(1 \mathrm{H}, \mathrm{m})$ \\
\hline 16 & $70.72 \mathrm{~d}$ & $4.14(1 \mathrm{H}, \mathrm{m})$ & 31 & $76.79 \mathrm{~d}$ & $3.04(1 \mathrm{H}, \mathrm{m})$ \\
\hline 17 & $55.44 \mathrm{~d}$ & $1.45(1 \mathrm{H}, \mathrm{m})$ & $4^{\prime}$ & $69.66 \mathrm{~d}$ & $3.24(1 \mathrm{H}, \mathrm{m})$ \\
\hline 18 & $19.39 \mathrm{q}$ & $1.12(3 \mathrm{H}, \mathrm{s})$ & $5^{\prime}$ & $65.62 \mathrm{t}$ & $\begin{array}{l}3.61(1 \mathrm{H}, \mathrm{m}) \\
2.97(1 \mathrm{H}, \mathrm{m})\end{array}$ \\
\hline
\end{tabular}

Table 2. Crystal data and structure refinement for riparsaponin.

\begin{tabular}{cc}
\hline Identification code & $070516 \mathrm{a}$ \\
Empirical formula & $\mathrm{C}_{36} \mathrm{H}_{57} \mathrm{O}_{8}$ \\
Formula weight & 617.82 \\
Temperature & $298(2) \mathrm{K}$ \\
Wave length & $0.71073 \mathrm{~A}$ \\
Crystal system, space group & Orthorhombic, P2(1)2(1)2(1) \\
& $\mathrm{a}=6.3405(9) \mathrm{Aalpha}=90 \mathrm{deg}$. \\
Unit cell dimensions & $\mathrm{b}=12.7265(17) \mathrm{Abeta}=90 \mathrm{deg}$. \\
& $\mathrm{c}=41.573(6) \mathrm{Agamma}=90 \mathrm{deg}$. \\
Volume & $3354.6(8) \mathrm{A}^{3}$ \\
Z, Calculated density & $4,1.223 \mathrm{Mg} / \mathrm{m}^{3}$ \\
Absorption coefficient & $0.085 \mathrm{~mm} \mathrm{~m}^{-1}$ \\
F (000) & 1348 \\
Crystalsize & $0.26 \times 0.22 \times 0.08 \mathrm{~mm}$ \\
The tarange for data collection & 1.67 to $28.31 \mathrm{deg}$. \\
\hline
\end{tabular}


Table 2. Cont.

\begin{tabular}{cc}
\hline Limiting indices & $-8 \leq \mathrm{h} \leq 8,-16 \leq \mathrm{k} \leq 16,-55 \leq 1 \leq 53$ \\
Reflections collected/unique & $29112 / 8026[\mathrm{R}(\mathrm{int})=0.0893]$ \\
Completeness to theta $=28.31$ & $98.9 \%$ \\
Absorption correction & MUTI-SCAN \\
Max. andmin. transmission & 1.000000 and 0.832723 \\
Refinement method & Full-matrixleast-squaresonF \\
Data/restraints/parameters & $8026 / 0 / 398$ \\
Goodness-of-fitonF ${ }^{2}$ & 0.786 \\
Final Rindices [I $>$ 2sigma(I) & $\mathrm{R}_{1}=0.0666, \mathrm{wR}_{2}=0.1877$ \\
Rindices (alldata) & $\mathrm{R}_{1}=0.1410, \mathrm{wR}_{2}=0.2517$ \\
Absolute structure parameter & $0.7(17)$ \\
Extinction coefficient & $0.0040(15)$ \\
Largest diff. Peak and hole & 0.342 and $-0.329 \mathrm{eA}^{-3}$ \\
\hline
\end{tabular}

\subsection{Inhibitory Effect of Riparsaponin on Xanthine Oxidase Activity in Vitro}

Gout is one of the common human metabolic diseases and caused by hyperuricemia, which can result in depositions of urate crystals in joints, leading to gouty arthritis [7]. Xanthine oxidase plays an important role during the formation of uric acid, and the accumulation of uric acid can result in hyperuricaemia, leading to gout [8]. Previous investigations revealed that inhibitors of xanthine oxidase could be potentially beneficial for treating gouty arthritis [9]. Herbal remedies have been used in China for more than millennium, and lots of investigations have reported that the herbal medicines and its derived compounds can safely and effectively in treatment of various diseases [10,11]. In our present study, the inhibitory activities of the six known compounds on xanthine oxidase were weak, but riparsaponin could significantly inhibit xanthine oxidase activity in vitro at the doses during 9.68 to $161.29 \mathrm{nmol} / \mathrm{mL}$ compared with the DMSO group $(p<0.01)$, in a dose-dependent manner (Table 3 ). In our present study, the $\mathrm{IC}_{50}$ of riparsaponin was $11.16 \mathrm{nmol} / \mathrm{mL}$, which is a better value compared to allopurinol used as positive control drug ( $\left.\mathrm{IC}_{50} 11.84 \mathrm{nmol} / \mathrm{mL}\right)$. The results above indicated that riparsaponin is a potential powerful xanthine oxidase inhibitor.

Table 3. Inhibitory effect of riparsaponin on xanthine oxidase activity $(n=5)$.

\begin{tabular}{|c|c|c|c|c|}
\hline Group & $\begin{array}{c}\text { Concentration } \\
(\mathbf{n m o l} / \mathbf{m L})\end{array}$ & $\begin{array}{c}\text { Fluorescence } \\
\text { Unit }\end{array}$ & $\begin{array}{l}\text { Inhibition } \\
\text { Ratio (\%) } \\
\end{array}$ & $\begin{array}{l}\text { IC }{ }_{50} \text { Value } \\
\text { (nmol/mL) }\end{array}$ \\
\hline DMSO & & $8.291 \pm 0.892$ & 0.07 & \\
\hline \multirow{6}{*}{ Riparsaponin } & 4.84 & $7.205 \pm 0.865$ & 13.09 & \multirow{6}{*}{11.16} \\
\hline & 9.68 & $4.815 \pm 0.861 * *$ & 41.93 & \\
\hline & 19.36 & $3.248 \pm 0.912 * *$ & 60.82 & \\
\hline & 40.32 & $2.362 \pm 0.127 * *$ & 71.52 & \\
\hline & 80.65 & $1.872 \pm 0.483 * *$ & 77.42 & \\
\hline & 161.29 & $1.474 \pm 0.131 * *$ & 82.22 & \\
\hline \multirow{6}{*}{ Allopurinol } & 2.20 & $6.796 \pm 0.696 *$ & 18.03 & \multirow{6}{*}{11.84} \\
\hline & 4.41 & $6.212 \pm 0.580 * *$ & 25.08 & \\
\hline & 8.82 & $4.879 \pm 0.410 * *$ & 40.93 & \\
\hline & 17.63 & $2.776 \pm 0.217 * *$ & 66.52 & \\
\hline & 35.27 & $2.062 \pm 0.370 * *$ & 75.13 & \\
\hline & 70.53 & $1.617 \pm 0.220 * *$ & 80.49 & \\
\hline
\end{tabular}

Data were expressed as Mean $\pm \mathrm{SD},{ }^{*} p<0.05,{ }^{* *} p<0.05$, compared to the DMSO group. 


\section{Experimental Section}

\subsection{General Information}

These following instruments were used: UV visible spectrophotometer (UV-1600) was made by Rayleigh Analytical Instrument Company (Beijing, China). Mass spectrometer (EI-MS) (VGAutoSpec-3000, Beckman, CA, USA). The NMR (AV-400, AV-500) and X-ray single crystal diffractometer (APEX II DUO) instruments were both made by Bruker (Bremen, Germany).

\subsection{Plant Material}

The stems of H. riparia were collected in Jinping County, Yunnan Province, China, in October 2010. The plant was identified by Jingxiu Li (Kunming Institute of Botany Chinese Academy of Science, Kunming, Yunnan, China). A voucher specimen was deposited in our laboratory.

\subsection{Extraction and Isolation}

Dried stems of $H$. riparia $(58.8 \mathrm{~kg}$ ) were powered and extracted three times with $60 \%$ ethanol (total $300 \mathrm{~L})$ at reflux. Then the extracts were spray-dried to obtain a dry fine powder $(4.8 \mathrm{~kg})$. The powder was extracted three times by maceration with petroleum ether, $\mathrm{CH}_{2} \mathrm{Cl}_{2}$, EtOAc, and $95 \%$ ethanol, respectively (total $40 \mathrm{~L}$, each extraction lasted 2 days).

After concentration, compound $\mathbf{2}$ was crystallized from the petroleum ether fraction, and the pure compound 2 (3.17 g) was obtained by recrystallization from EtOAc. Then, the petroleum ether fraction (73 g) was subjected to column chromatography (CC) over silica gel (200-300 mesh) eluting with petroleum ether-EtOAc $(9: 1,8: 1,5: 1,3: 1,1: 1,1: 3,1: 5,1: 9)$, and seven sub-fractions $\mathbf{A}-\mathbf{G}$ were obtained on the basis of TLC analysis. Compound $\mathbf{4}$ was crystallized from fraction $\mathbf{B}$, and the pure compound $4(60 \mathrm{mg})$ was obtained by recrystallization from petroleum ether- $\mathrm{CH}_{2} \mathrm{Cl}_{2}$ (9:1). Fraction $\mathbf{C}$ was subjected to $\mathrm{CC}$ over silica gel (200-300 mesh) eluting with petroleum ether- $\mathrm{CH}_{2} \mathrm{Cl}_{2}(9: 1,8: 1$, $5: 1,3: 1,1: 1,1: 3,1: 5,1: 9)$, and combination of similar fractions after TLC comparison afforded five fractions $\mathbf{C}_{\mathbf{1}}-\mathbf{C}_{\mathbf{5}}$. Compound $\mathbf{5}$ was crystallized from fraction $\mathbf{C}_{\mathbf{2}}$, and the pure compound $\mathbf{5}(87 \mathrm{mg})$ was obtained by recrystallization from EtOAc.

The $\mathrm{CH}_{2} \mathrm{Cl}_{2}$ fraction (100 g) was subjected to AB-8 macroporous resin (The Chemical Plant of Nankai University, Tianjing, China) CC eluting with gradient ethanol (0\%, 30\%, 70\%, 90\%), and four fractions $\mathbf{A}-\mathbf{D}$ were obtained. After concentration, compound $\mathbf{3}$ was crystallized from fraction $\mathbf{D}$, and the pure compound $3(45 \mathrm{mg})$ was obtained by recrystallization in petroleum ether-EtOAC $(9: 1)$. Fraction $\mathbf{C}$ was subjected to $\mathrm{CC}$ over silica gel (200-300 mesh) eluting with petroleum ether-acetone (9:1, 8:1, 5:1, 3:1, 1:1, 1:3), and four sub-fractions $\mathbf{C}_{\mathbf{1}}, \mathbf{C}_{2}, \mathbf{C}_{\mathbf{3}}, \mathbf{C}_{\mathbf{4}}$ were obtained on the basis of TLC. Sub-fraction $\mathbf{C}_{2}$ was subjected to $\mathrm{CC}$ over silica gel (200-300 mesh) eluting with petroleum ether-acetone (9:1, 8:1, 5:1, 3:1, 1:1, 1:3) again to afford four sub-fractions $\mathbf{C}_{\mathbf{2 1}}-\mathbf{C}_{\mathbf{2 4}}$, and compound $\mathbf{6}$ (white powder, $144 \mathrm{mg}$ ) was separated out from fraction $\mathbf{C}_{\mathbf{2 2}}$.

The EtOAc fraction (72.3 g) was subjected to CC over silica gel (200-300 mesh) eluting with petroleum ether-acetone $(15: 1,10: 1,7: 1,5: 1,3: 1,1: 1,1: 2)$, to give six sub-fractions $\mathbf{A}-\mathbf{F}$ on the basis of TLC analysis. After concentration, compound 7 (a white powder, $24 \mathrm{mg}$ ) was separated out from 
fraction E. Fraction B was repeatedly subjected to CC over silica gel (200-300 mesh) eluting with petroleum ether-acetone $(10: 1,7: 1,5: 1,3: 1,1: 1,1: 2)$, and four sub-fractions $\mathbf{B}_{1}-\mathbf{B}_{4}$ of $\mathbf{B}$ were thus obtained. Then sub-fraction $\mathbf{B}_{\mathbf{2}}$ was subjected to $\mathrm{CC}$ over silica gel again eluting with a gradient of petroleum ether-acetone, and five fractions $\mathbf{B}_{\mathbf{2 1}}-\mathbf{B}_{\mathbf{2 5}}$ were obtained; then compound $\mathbf{1}$ was crystallized from $\mathbf{B}_{\mathbf{2 2}}$, and the pure compound $\mathbf{1}$ (213 $\mathrm{mg}$ ) was obtained by recrystallization from petroleum ether-EtOAC.

2,4-Sorbic acid (2) was obtained as colorless crystals (EtOAc), mp: $134-136{ }^{\circ} \mathrm{C}$; EI-MS $(\mathrm{m} / \mathrm{z})(\%)$ : 111 (38), 97 (71), 83 (80), 69 (90), 57 (100); ${ }^{1} \mathrm{H}-\mathrm{NMR}$ (400 MHz, CDCl 3 , ppm) $\delta: 5.75$ (1H, d, $J=15.3 \mathrm{~Hz}, \mathrm{H}-2), 7.33$ (1H, dd, $J=15.4,9.7 \mathrm{~Hz}, \mathrm{H}-3), 6.26(1 \mathrm{H}, \mathrm{m}, \mathrm{H}-4), 5.79$ (1H, s, H-5), 1.87 (3H, $\left.\mathrm{d}, J=5.0 \mathrm{~Hz}, \mathrm{CH}_{3}-6\right) ;{ }^{13} \mathrm{C}-\mathrm{NMR}\left(100 \mathrm{MHz}, \mathrm{CDCL}_{3}, \mathrm{ppm}\right) \delta: 173.5$ (C-1), 118.4 (C-2), 147.7 (C-3), 130.0 (C-4), 141.2 (C-5), 19.1 (C-6).

Ethyl gallate (3) was obtained as colorless crystals [petroleum ether-EtOAc (9:1)], mp: $169-170{ }^{\circ} \mathrm{C}$; FAB-MS (m/z) (\%): 197 (90), 169 (5), 154 (100), 124 (3), 97 (3), 80 (15), 64 (3); ${ }^{1} \mathrm{H}-\mathrm{NMR}$ (400 MHz, $\left.\mathrm{CD}_{3} \mathrm{ODCD}_{3}, \mathrm{ppm}\right) \delta: 7.11(2 \mathrm{H}, \mathrm{s}, \mathrm{H}-2, \mathrm{H}-6), 4.26\left(2 \mathrm{H}, \mathrm{q}, J=7.0 \mathrm{~Hz}, \mathrm{OCH}_{2} \mathrm{CH}_{3}\right), 1.27(3 \mathrm{H}, \mathrm{t}, J=7.2 \mathrm{~Hz}$, $\left.\mathrm{OCH}_{2} \mathrm{CH}_{3}\right), 6.94(2 \mathrm{H}, \mathrm{d}, J=1.0 \mathrm{~Hz}), 8.12(1 \mathrm{H}, \mathrm{s}), 9.25(2 \mathrm{H}, \mathrm{s}) ;{ }^{13} \mathrm{C}-\mathrm{NMR}\left(100 \mathrm{MHz}, \mathrm{CD}_{3} \mathrm{ODCD}_{3}\right.$, ppm) $\delta: 122.0$ (C-1), 109.7 (C-2), 146.0 (C-3), 138.6 (C-4), 146.0 (C-5), 109.7 (C-6), 166.6 (C-7), 60.9 (C-8), 14.6 (C-9).

Acetylaleuritolic acid (4) was obtained as colorless crystals [petroleum ether- $\mathrm{CH}_{2} \mathrm{Cl}_{2}$ (9:1)], mp: 287-288 ${ }^{\circ} \mathrm{C}$; EI-MS (m/z) (\%): $498\left(\mathrm{M}^{+}, 3\right), 439\left(\mathrm{M}^{+}-\mathrm{COCH}_{3}, 2\right), 344$ (RDA fragment, 5), 285 (344- $\left.\mathrm{COCH}_{3}\right), 189(100) .{ }^{1} \mathrm{H}-\mathrm{NMR}\left(400 \mathrm{MHz}, \mathrm{CDCl}_{3}, \mathrm{ppm}\right) \delta: 0.75\left(3 \mathrm{H}, \mathrm{s}, \mathrm{CH}_{3}-26\right), 0.84(3 \mathrm{H}, \mathrm{s}$, $\left.\mathrm{CH}_{3}-23\right), 0.88$ (3H, s, $\left.\mathrm{CH}_{3}-24\right), 0.91\left(3 \mathrm{H}, \mathrm{s}, \mathrm{CH}_{3}-29\right), 0.93$ (3H, s, $\left.\mathrm{CH}_{3}-30\right), 0.95\left(3 \mathrm{H}, \mathrm{s}, \mathrm{CH}_{3}-25\right), 1.13$ $\left(3 \mathrm{H}, \mathrm{s}, \mathrm{CH}_{3}-27\right), 2.04\left(3 \mathrm{H},-\mathrm{COCH}_{3}\right), 4.48(1 \mathrm{H}, \mathrm{t}, J=8.0 \mathrm{~Hz}, \mathrm{H}-3), 5.50(1 \mathrm{H}, \mathrm{t}, J=3.3 \mathrm{~Hz}, \mathrm{H}-15)$; ${ }^{13} \mathrm{C}-\mathrm{NMR}\left(100 \mathrm{MHz}, \mathrm{CDCl}_{3}, \mathrm{ppm}\right) \delta: 37.9$ (C-1), 22.4 (C-2), 80.9 (C-3), 37.7 (C-4), 55.3 (C-5), 18.1 (C-6), 33.6 (C-7), 39.0 (C-8), 47.5 (C-9), 37.0 (C-10), 23.4 (C-11), 32.4 (C-12), 41.3 (C-13), 160.5 (C-14), 116.8 (C-15), 23.5 (C-16), 46.5 (C-17), 41.3 (C-18), 45.8 (C-19), 30.6 (C-20), 32.4 (C-21), 27.9 (C-22), 28.6 (C-23), 16.6 (C-24), 15.6 (C-25), 17.3 (C-26), 25.9 (C-27), 184.2 (C-28), 33.0 (C-29), $23.6(\mathrm{C}-30), 171.0(-\mathrm{COOH}), 21.3\left(-\mathrm{COCH}_{3}\right)[12]$.

Spinasterol (5) was obtained as colorless crystals $\left(\mathrm{CH}_{2} \mathrm{Cl}_{2}\right), \mathrm{mp}: 162-163{ }^{\circ} \mathrm{C}$; EI-MS $(\mathrm{m} / \mathrm{z})(\%): 412$ $\left(\mathrm{M}^{+}\right), 396\left(\mathrm{M}^{+}-\mathrm{O}\right), 351(369-\mathrm{OH}), 271\left(300-\mathrm{C}_{2} \mathrm{H}_{5}\right), 255$ (271-O), $213\left(255-\mathrm{C}_{3} \mathrm{H}_{6}\right) ;{ }^{1} \mathrm{H}-\mathrm{NMR}(400 \mathrm{MHz}$, $\left.\mathrm{CDCl}_{3}, \mathrm{ppm}\right) \delta: 5.15(1 \mathrm{H}, \mathrm{H}-7), 5.03(1 \mathrm{H}, \mathrm{dd}, J=8.6 \mathrm{~Hz}, \mathrm{H}-22), 5.21(1 \mathrm{H}, \mathrm{dd}, J=8.5 \mathrm{~Hz}, \mathrm{H}-23), 0.70$ $\left(3 \mathrm{H}, \mathrm{s}, \mathrm{CH}_{3}-18\right), 0.81\left(3 \mathrm{H}, \mathrm{s}, \mathrm{CH}_{3}-19\right), 0.83\left(3 \mathrm{H}, \mathrm{t}, J=7.3 \mathrm{~Hz}, \mathrm{CH}_{3}-29\right), 0.85$ (3H, d, $J=6.3 \mathrm{~Hz}$, $\left.\mathrm{CH}_{3}-26\right), 0.86\left(3 \mathrm{H}, \mathrm{d}, J=6.3 \mathrm{~Hz}, \mathrm{CH}_{3}-26\right), 1.04\left(3 \mathrm{H}, \mathrm{d}, J=6.6 \mathrm{~Hz}, \mathrm{CH}_{3}-21\right), 3.54(\mathrm{H}, \mathrm{tt}, J=11.0 \mathrm{~Hz}$, $\mathrm{H}-3)$; ${ }^{13} \mathrm{C}-\mathrm{NMR}\left(100 \mathrm{MHz}, \mathrm{CDCl}_{3}, \mathrm{ppm}\right)$ ): 37.2 (C-1), 31.6 (C-2), 71.8 (C-3), 37.9 (C-4), 40.5 (C-5), 29.6 (C-6), 121.7 (C-7), 140.7 (C-8), 50.1 (C-9), 36.1 (C-10), 21.2 (C-11), 39.6 (C-12), 42.5 (C-13), 55.9 (C-14), 23.0 (C-15), 28.9 (C-16), 56.8 (C-17), 12.0 (C-18), 19.0 (C-19), 42.2 (C-20), 21.0 (C-21), 138.3 (C-22), 129.2 (C-23), 51.2 (C-24), 31.9 (C-25), 21.1 (C-26), 19.4 (C-27), 25.4 (C-28), 12.2 (C-29) [13].

Daucosterol (6) was obtained as white powders, mp: $288-289{ }^{\circ} \mathrm{C} ;{ }^{1} \mathrm{H}-\mathrm{NMR}\left(400 \mathrm{MHz}, \mathrm{C}_{5} \mathrm{D}_{5} \mathrm{~N}\right.$, ppm) $\delta: 5.17(1 \mathrm{H}, \mathrm{m}, J=5.2 \mathrm{~Hz}, \mathrm{H}-6), 4.32(1 \mathrm{H}, \mathrm{d}, J=7.2 \mathrm{~Hz}, \mathrm{H}-1$ '), 4.07 (1H, m, H-3), 0.75 (3H, d, 
$\left.J=8.0 \mathrm{~Hz}, \mathrm{CH}_{3}-21\right), 0.70\left(3 \mathrm{H}, \mathrm{s}, \mathrm{CH}_{3}-19\right), 0.65\left(6 \mathrm{H}, \mathrm{d}, J=8.4 \mathrm{~Hz}, \mathrm{CH}_{3}-26,27\right), 0.59(3 \mathrm{H}, \mathrm{t}, J=4.0 \mathrm{~Hz}$, $\left.\mathrm{CH}_{3}-29\right), 0.42\left(3 \mathrm{H}, \mathrm{s}, \mathrm{CH}_{3}-18\right) ;{ }^{13} \mathrm{C}-\mathrm{NMR}\left(100 \mathrm{MHz}, \mathrm{C}_{5} \mathrm{D}_{5} \mathrm{~N}, \mathrm{ppm}\right) \delta: 37.5(\mathrm{C}-1), 32.0(\mathrm{C}-2), 78.6$ (C-3), 39.9 (C-4), 140.9 (C-5), 121.9 (C-6), 32.2 (C-7), 32.0 (C-8), 50.3 (C-9), 36.4 (C-10), 21.3 (C-11), 39.3 (C-12), 42.5 (C-13), 56.8 (C-14), 23.4 (C-15), 27.3 (C-16), 56.2 (C-17), 12.0 (C-18), 19.4 (C-19), 36.4 (C-20), 19.0 (C-21), 34.2 (C-22), 26.3 (C-23), 45.8 (C-24), 29.4 (C-25), 19.2 (C-26), 20.0 (C-27), 22.8 (C-28), 12.0 (C-29), 102.6 (C-1'), 75.3 (C-2'), 78.5 (C-3'), 71.7 (C-4'), 78.0 (C-5'), 62.8 (C-6').

Taraxerone (7) was obtained as a white powder, mp: $288-289{ }^{\circ} \mathrm{C}$; EI-MS $(\mathrm{m} / \mathrm{z})(\%): 424\left(\mathrm{M}^{+}, 23\right), 409$ $\left(\mathrm{M}^{+}-\mathrm{CH}_{3}, 17\right), 300$ (RDA fragment, 80), 285 (70), 272 (19), 257 (20), 205 (C ring fragment, 65), 204 (100), 189 (45), 133 (62); ${ }^{1} \mathrm{H}-\mathrm{NMR}\left(500 \mathrm{MHz}, \mathrm{CDCl}_{3}, \mathrm{ppm}\right) \delta: 0.82\left(3 \mathrm{H}, \mathrm{s}, \mathrm{CH}_{3}\right), 0.91\left(3 \mathrm{H}, \mathrm{s}, \mathrm{CH}_{3}\right)$, $0.95\left(3 \mathrm{H}, \mathrm{s}, \mathrm{CH}_{3}\right), 0.98\left(3 \mathrm{H}, \mathrm{s}, \mathrm{CH}_{3}\right), 1.08\left(3 \mathrm{H}, \mathrm{s}, \mathrm{CH}_{3}\right), 1.10\left(3 \mathrm{H}, \mathrm{s}, \mathrm{CH}_{3}\right), 1.13\left(3 \mathrm{H}, \mathrm{s}, \mathrm{CH}_{3}\right), 1.15(3 \mathrm{H}$, $\left.\mathrm{s}, \mathrm{CH}_{3}\right), 5.56(1 \mathrm{H}, \mathrm{dd}, J=3.2,8.2 \mathrm{~Hz}, \mathrm{H}-15),{ }^{13} \mathrm{C}-\mathrm{NMR}\left(125 \mathrm{MHz}, \mathrm{CDCl}_{3}, \mathrm{ppm}\right) \delta: 38.4(\mathrm{C}-1)$, 33.6 (C-2), 217.5 (C-3), 47.6 (C-4), 55.8 (C-5), 20.0 (C-6), 37.7 (C-7), 38.9 (C-8), 48.8 (C-9), 37.5 (C-10), 17.4 (C-11), 34.1 (C-12), 29.8 (C-13), 157.6 (C-14), 117.1 (C-15), 36.7 (C-16), 35.7 (C-17), 48.8 (C-18), 40.0 (C-19), 28.8 (C-20), 33.1 (C-21), 35.1 (C-22), 26.1 (C-23), 21.5 (C-24), 14.8 (C-25), 29.9 (C-26), 25.6 (C-27), 29.9 (C-28), 33.4 (C-29), 21.3 (C-30) [14].

\subsection{Xanthine Oxidase Inhibitory Assay}

The inhibitory effect of compound $\mathbf{1}$ against xanthine oxidase activity was carried out according to the previous investigations [15]. The experiment was performed by using 96 holes plate, and the allopurinol and DMSO were used as the positive and negative control. Firstly, xanthine $(50 \mu \mathrm{mol} / \mathrm{L})$ was added into each reaction system, and then the tested samples were added. After that, the xanthine oxidase $(0.1 \mathrm{U} / \mathrm{mL})$, quinoline solution $(5 \mu \mathrm{mol} / \mathrm{L}),\left(\mathrm{NH}_{4}\right)_{2} \mathrm{Fe}\left(\mathrm{SO}_{4}\right)$ solution $(1 \mu \mathrm{mol} / \mathrm{L}), \mathrm{H}_{2} \mathrm{SO}_{4}$ solution $(50 \mu \mathrm{mol} / \mathrm{L})$ were added subsequently. The reaction system incubated at room temperature for $18 \mathrm{~min}$, then added $\mathrm{NaOH}$ solution $(1 \mathrm{~mol} / \mathrm{L})$ into total reaction system to $150 \mu \mathrm{L}$. Finally, adding $150 \mu \mathrm{L}$ ethanol to terminate the reaction, and the reaction production was determined by measuring the absorbance at $380 \mathrm{~nm}$ by using fluorescent chemiluminescence detector on a microplate spectrophotometer. The inhibitory percentage against xanthine oxidase (\%) was calculated using the following equation:

$$
\text { inhibition }(\%)=[1-(\text { A sample } / \text { A control }) \times 100
$$

In addition, the $\mathrm{IC}_{50}$ values were calculated

\section{Conclusions}

In the present study, a new cycloartane-type triterpenoid saponin named riparsaponin was isolated from the stem of $H$. riparia, and it has a significant inhibitory effect on xanthine oxidase.

\section{Acknowledgments}

This work was supported by the Key Project of Basic Research and Application Program of Yunnan Province in China (No. 2011FA036) 


\section{Author Contributions}

Conceived and designed the experiments: JZ; performed the experiments: FX, XQZ, LLY, Analyzed the data: FX, XHW; wrote the paper: FX, JZ.

\section{Conflicts of Interest}

The authors declare no conflict of interest.

\section{References}

1. Chinese Academy of Medical Sciences Research Institute. Higher Plant Figure of China; Beijing Science Press: Beijing, China, 1972; Volume 2, pp. 606-608.

2. State Administration of Traditional Chinese Medicine. Chinese Material Medica; Science and Technology Press of Shanghai: Shanghai, China, 1999; Volume 6, pp. 824-825.

3. Lee, I.; Kim, J.; Kim, Y.S.; Yoo, N.H.; Kim, C.S.; Jo, K.; Kim, J.H.; Bach, T.T.; Kim, J.S. Cycloartane-type triterpenes from the leaves of Homonoia Riparia with VEGF-induced angiogenesis inhibitor activity. J. Nat. Prod. 2012, 75, 1312-1318.

4. Parver, N.; Pal-Singh, M.; Khan, N.U. Chemical examination of the leaves of Homonoia riparia Lour (Euphorbiaceae). J. Indian Chem. Soc. 1988, 65, 815-816.

5. Yang, S.M.; Liu, X.K.; Qing, C.; Wu, D.G.; Zhu, D.Y. Chemical constituents from the roots of Homonoia riparia. Acta Pharm. Sin. 2007, 42, 292-296.

6. Wu, L.J. Natural Pharmaceutical Chemistry, 5th ed.; People's Medical Publishing House: Beijing, China, 2004; pp. 93-103.

7. Sowndhararajan, K.; Joseph, J.M.; Rajendrakumaran, D. In vitro xanthine oxidase inhibitory activity of methanol extracts of Erythrina indica Lam. leaves and stem bark. Asian Pac. J. Trop. Biomed. 2012, 2, S1415-S1417.

8. Masuoka, N.; Nihei, K.; Maeta, A.; Yamagiwa, Y.; Kubo, I. Inhibitory effects of cardols and related compounds on superoxide anion generation by xanthine oxidase. Food Chem. 2014, 166, 270-274.

9. Sweeney, A.P.; Wyllie, S.G.; Shalliker, R.A.; Markham, J.L. Xanthine oxidase inhibitory activity of selected Australian native plants. J. Ethnopharmacol. 2001, 75, 273-277.

10. Peng, W.; Ming, Q.L.; Han, P.; Zhang, Q.Y.; Jiang, Y.P.; Zheng, C.J.; Han, T.; Qin, L.P. Anti-allergic rhinitis effect of caffeoylxanthiazonoside isolated fromfruits of Xanthium strumarium L. in rodent animals. Phytomedicine 2014, 21, 824-829.

11. Karki, R.; Park, C.H.; Kim, D.W. Extract of buckwheat sprouts scavenges oxidation and inhibits pro-inflammatory mediators in lipopolysaccharide-stimulated macrophages (RAW264.7). J. Integr. Med. 2013, 11, 246-252.

12. Nyasse, B.; Ngantchou, I.; Nono, J.J.; Schneider, B. Antifilarial activity in vitro of polycarpol and 3-O-acetyl aleuritolic acid from cameroonian medicinal plants against Onchocerca gutturosa. Nat. Prod. Res. 2006, 20, 391-397.

13. Villasenor, I.M.; Domingo, P.D. Antieareinogenicity potential of spinasterol isolated from squash flowers. Teratog. Carcinog. Mutagen. 2000, 20, 99-105. 
14. Wang, G.K.; Lin, B.B.; Qin, M.J. Study on chemical constituents from leaf of Bombax ceiba (II). J. Chin. Med. Mater. 2014, 37, 240-242.

15. Tsai, S.F.; Lee, S.S. Neolignans as xanthine oxidase inhibitors from Hyptis rhomboids. Phytochemistry 2014, 101, 121-127.

Sample Availability: Contact the authors.

(C) 2014 by the authors; licensee MDPI, Basel, Switzerland. This article is an open access article distributed under the terms and conditions of the Creative Commons Attribution license (http://creativecommons.org/licenses/by/3.0/). 